\title{
Traitement et exploration du fichier Log du serveur web, pour l'extraction des connaissances : Web usage mining
}

\author{
Mostafa Hanoune* et Faouzia Benabbou
}

Laboratoire des technologies de l'information et modélisation (TIM), Faculté des sciences Ben M'Sik, Université Hassan II, Mohammedia, Casablanca, Maroc

(Reçu le 10 Juillet 2006, accepté le 19 Septembre 2006)

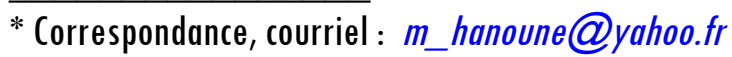

\section{Résumé}

L'objectif de ce travail est la conception et la réalisation d'un outil logiciel, en utilisant les concepts du "Web usage mining II, qui permettra au "Webmaster " d'avoir l'ensemble des connaissances sur le site web qu'il gère, en vue d'une amélioration et personnalisation.

II s'agit en fait, d'extraire de l'information à partir du fichier Log du serveur Web, hébergeant le site Web, et prendre les décisions pour découvrir les habitudes des internautes, et de répondre à leurs besoins en adaptant le contenu, la forme et l'agencement des pages web.

Mots-clés : "Web usage mining ", extraction de connaissances, fichier Log, méthode "I A Priori I, algorithme, théorie de décision.

\section{Abstract}

Log file treatment and exploration of the web site, for extracting and mining the knowledges : Web usage mining

The aim of this work is to design and produce one tool and implement, base on KDD (Knowledge discoverer on data base), by using the concept of Web usage mining, to give to the Webmasters, collections of Knowledge, including the statistics on site, in order to take the favourable decisions.

We will use the "Log file" on the Web server, accommodating the site Web, to discover the habits of users and respond to there requirements and needs, the shape and layout of Web pages and the contain generally speaking, with waiting of users.

Keywords : Web usage mining, extraction of knowledge, log file, "IA Priorill method, decision theory. 


\section{Introduction}

L'activité sur le Web et les données résultantes ont connu une croissance très rapide, vu la croissance exponentielle du nombre des documents mis en ligne. D'après des statiques sur des sites spécialisés, le nombre des utilisateurs d'Internet dans le monde a dépassé le milliard (1 076203 987), au mois de novembre 2006 [1], et le nombre de sites Web a atteint (105 244 649), au mois de décembre 2006 [2]. Ces données, en particulier celles relatives à l'usage du Web, sont traitées dans le Web Usage Mining (WUM) [3]. Dans cet article, nous nous intéressons à l'analyse des fichiers "I Log " [4-5], afin de comprendre le comportement des internautes sur un site Web ( Site de l'université Hassan IIMohammedia du Maroc: www.univh2m.ac.ma).

L'apport de ce travail réside principalement dans les points suivants :

\section{1-1. Connaissances sur les visiteurs}

Détenir le pourcentage des visiteurs par semaine, par mois et par an, Avoir une visibilité internationale : d'où proviennent les visiteurs?

\section{1-2. Connaissances sur les pages}

Reconnaître les pages Web les plus et les moins consultées (pages populaires et pages impopulaires),

Connaître les combinaisons des pages consultées,

Savoir quels sont les liens qui référencent le mieux.

\section{1-3. Connaissances sur les navigateurs et les U OS ॥}

Connaître le pourcentage des navigateurs les plus utilisés, Connaître également le pourcentage des systèmes d'exploitations les plus utilisés,

Le présent article est donc subdivisé en trois sections distinctes:

- La première section présente la conception, par la méthode UML, de la solution mise en place.

- Dans la deuxième section, on trouve les différentes étapes du prétraitement et nettoyage du fichier $\log$.

- La dernière est consacrée à l'exploration et l'analyse du fichier Log. 


\section{Analyse du problème et conception de la solution méthode UML}

\section{2-1. Diagramme de cas d'utilisation}

Dans cette étape, il s'agira de structurer les besoins des utilisateurs et les objectifs correspondants.

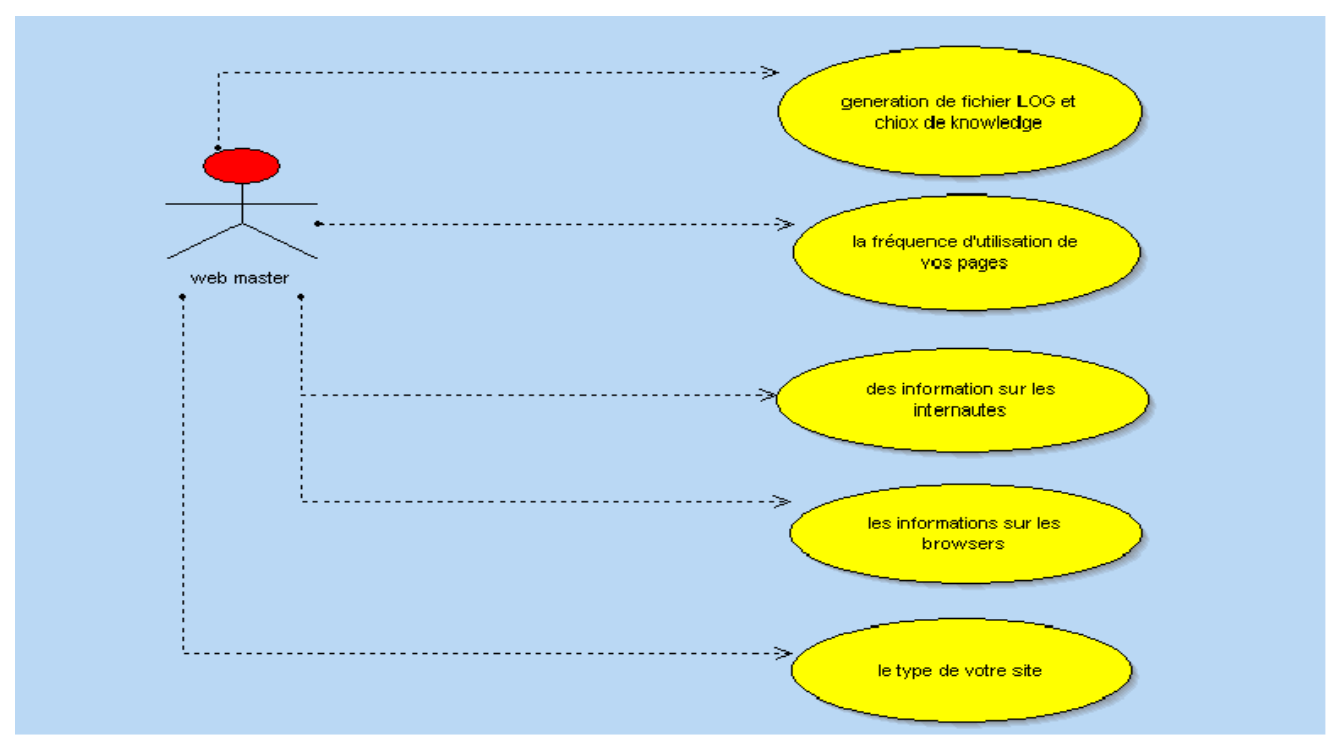

Figure 1 : Cas d'utilisation

2-2. Diagramme de classe

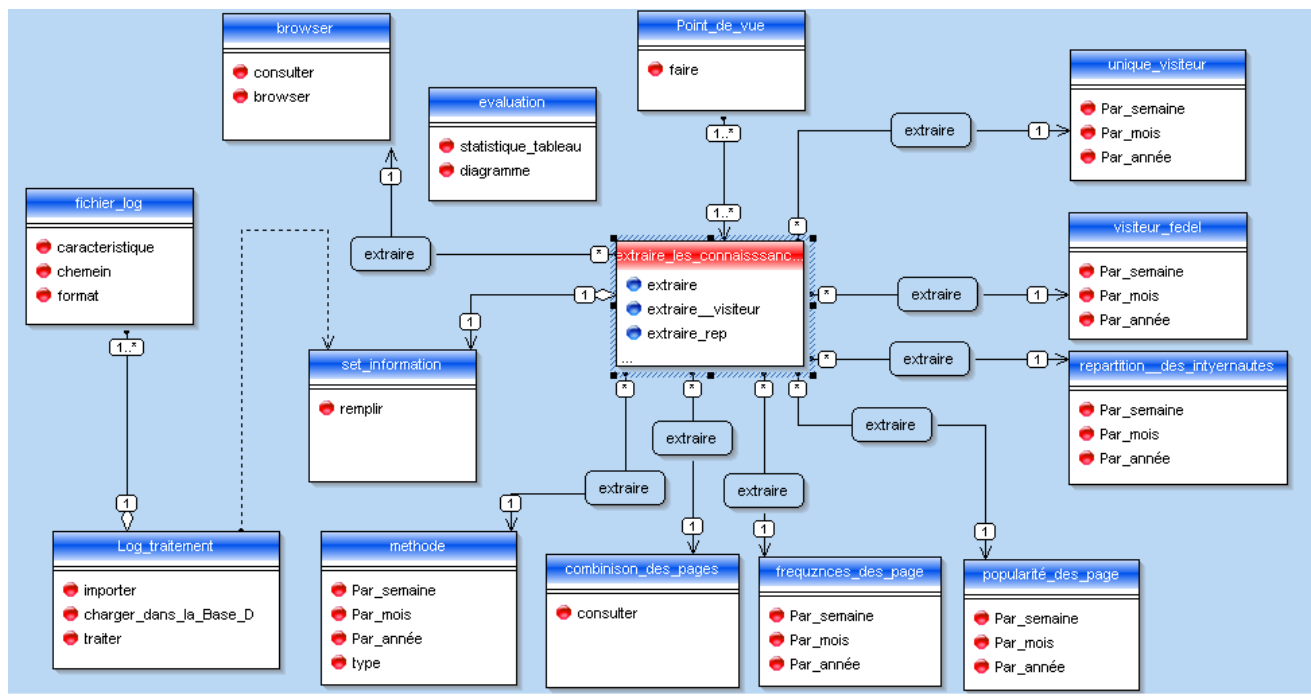

Figure 2 : Diagramme des classes 
A la Figure 1, le cercle en rouge signifie que c'est une fonction. On utilisera le diagramme de classe pour montrer la structuration du modèle à adopter, en mettant en évidence les différentes associations et lien entre les classes.

\section{2-3. Diagramme d'état de transition}

\section{2-3-1. DET de traitement de fichier LOG}

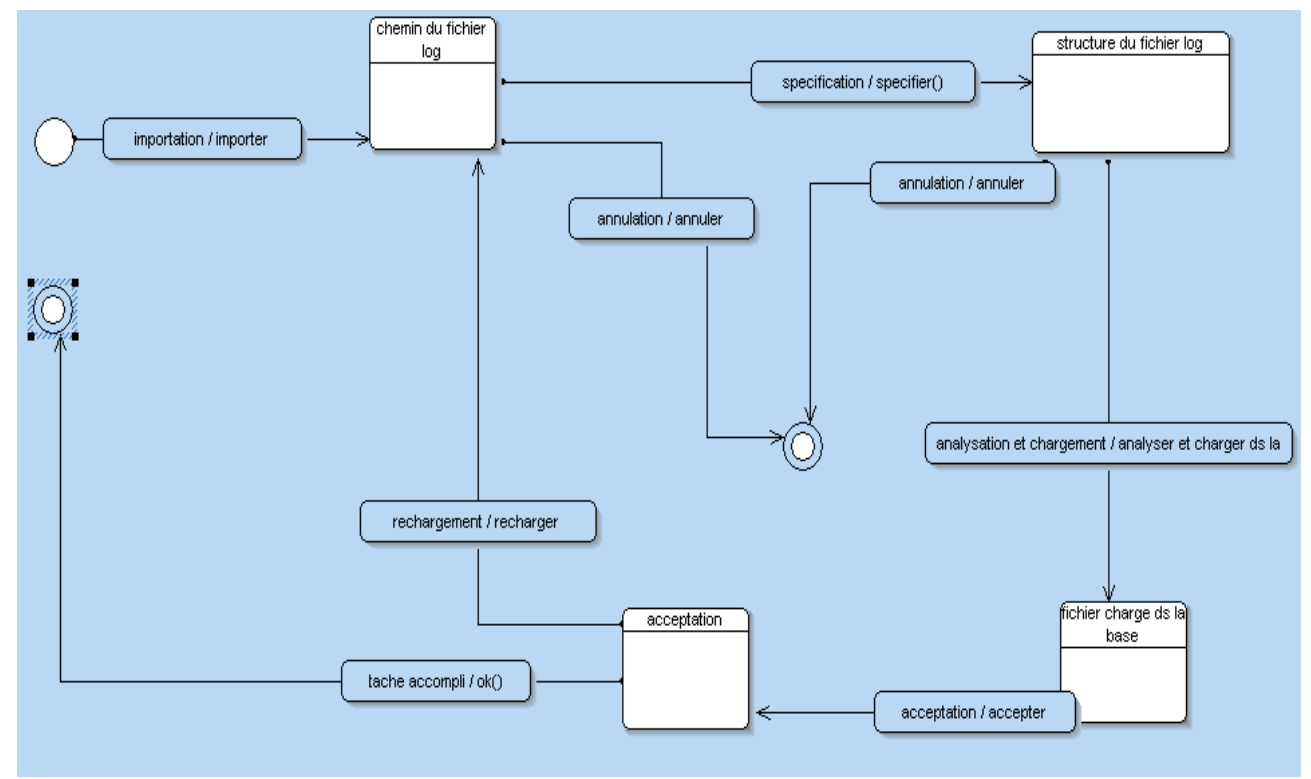

Figure 3 : Etats de transition

Le diagramme d'état de transitions nous permettra de décrire les changements d'états d'un objet ou d'un composant, en réponse aux interactions avec d'autres objets/composants ou avec des acteurs.

\section{2-3-2. DET extraction connaissance}

On montrera le processus suivi pour aboutir aux connaissances souhaitées. 


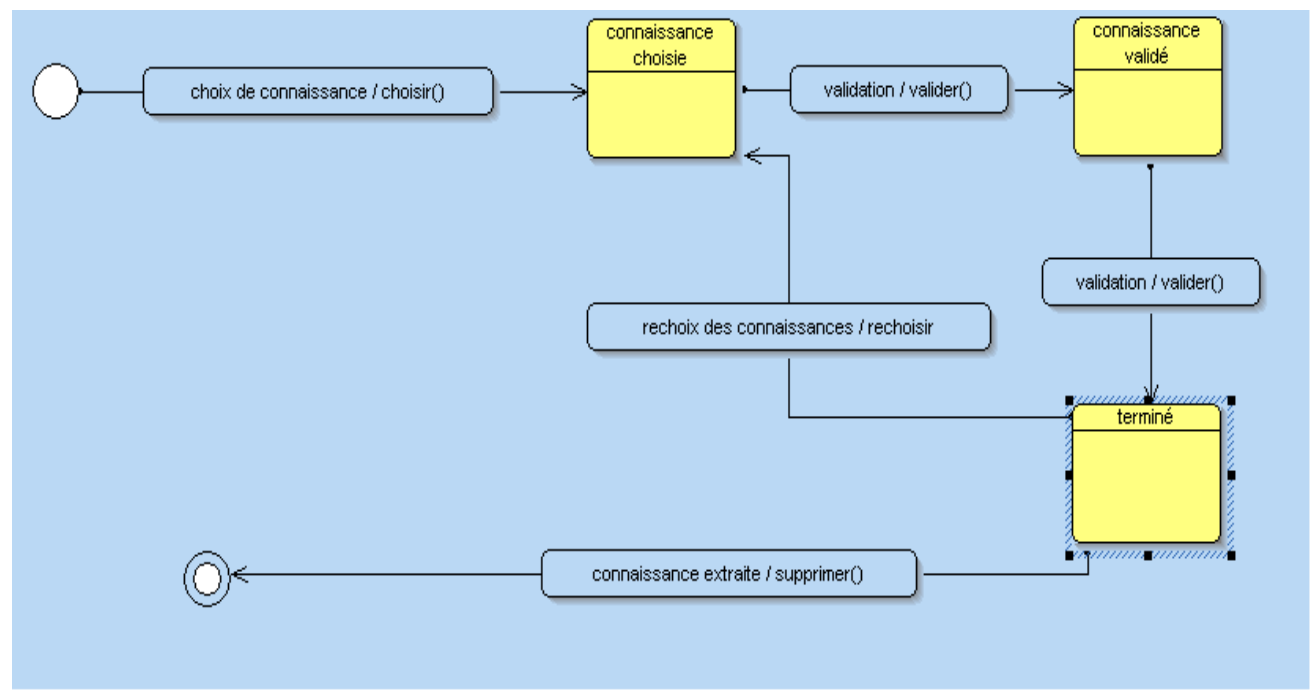

Figure 4 : Diagramme d'extraction de la connaissance

\section{2-4. Diagramme de séquences}

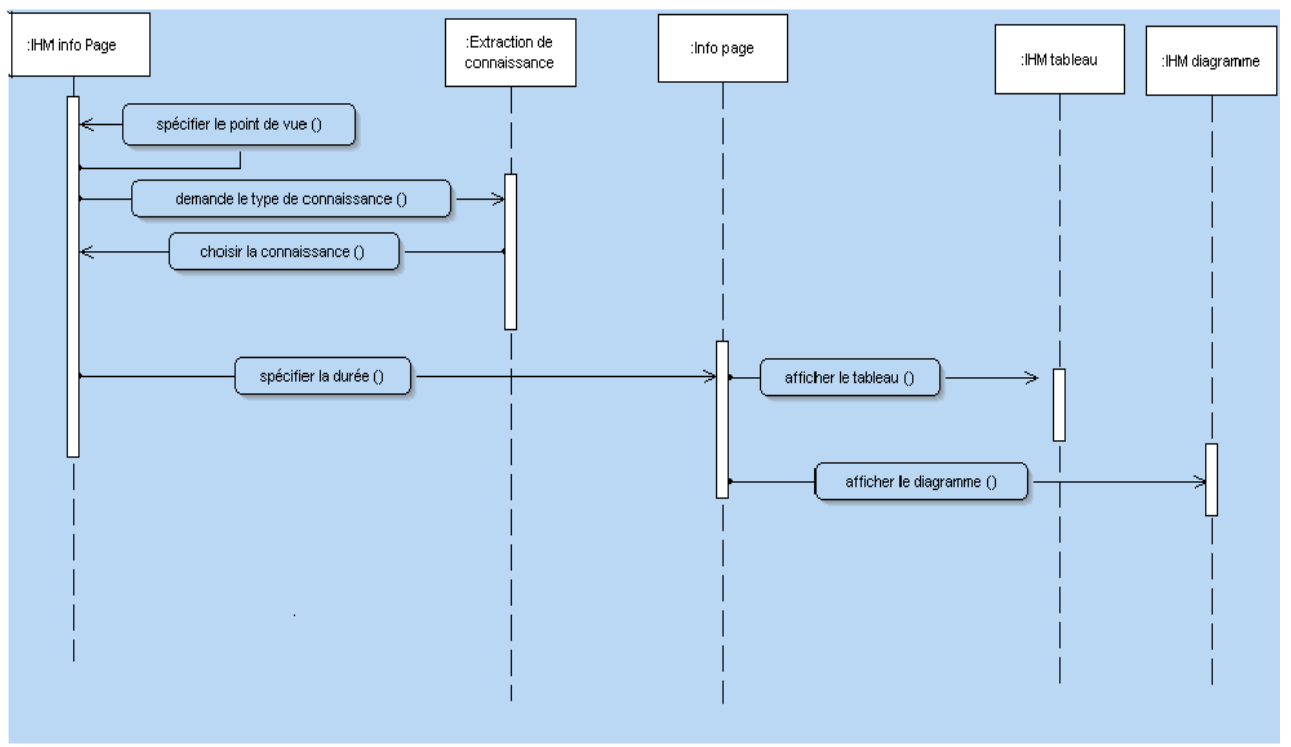

Figure 5 : Séquences d'utilisation

Le diagramme de séquences permet de représenter des collaborations entre objets selon un point de vue temporel, on y met l'accent sur la chronologie des envois de messages. 


\section{Prétraitement et nettoyage du fichier Log}

\section{3-1. Chargement du fichier Log et transformation en une table d'une BDD}

Le fichier $L O G$ est un fichier Texte, appelé aussi journal des connexions, qui conserve les traces des requêtes et des opérations traitées par le serveur. Généralement il est de la forme suivante, pour laquelle les différents champs de ce fichier seront importés dans une base de données définie comme suit :

- Le fichier $\log$ se transforme en une table composée de plusieurs colonnes. Chaque colonne correspond à un champ spécifique du fichier $\log$,

- La colonne II hote_client II correspond aux adresses IP des visiteurs,

- La colonne II login_client ॥ correspond au Nom du serveur utilisé par le visiteur,

- La colonne II utilisateur_client II correspond au Nom de l'utilisateur (en cas d'accès par mot de passe),

- La colonne II date_et_heure II correspond à la date d'accès,

- La colonne II methode II correspond à la méthode utilisée (GET/POST),

- La colonne II url_des_pages II correspond au URL demandé,

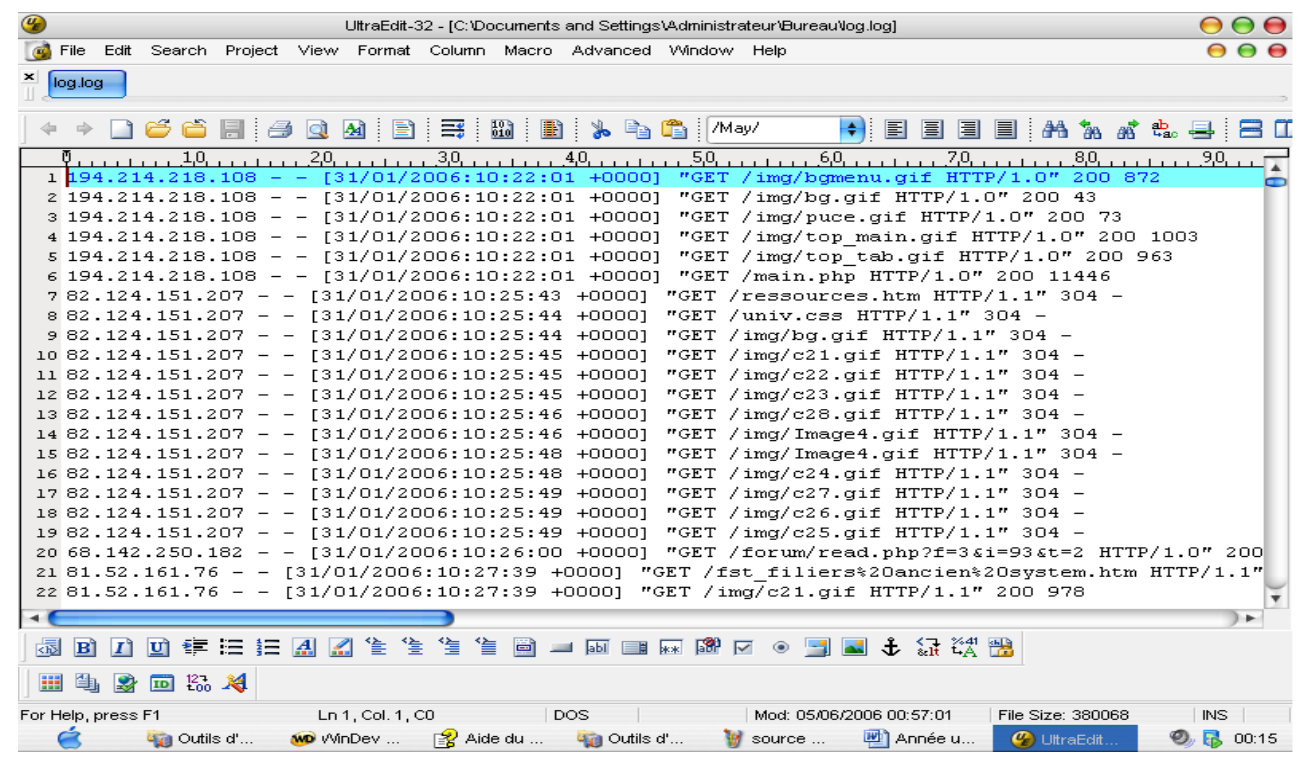

Figure 6 : Fichier $\log$ brut

- La colonne II protocole II correspond au protocole utilisé,

- La colonne II code_de_retour II,

- La colonne II taille_chargé || correspond à la taille chargée, ce fichier doit impérativement être pré-traité et nettoyé [6]. 


\begin{tabular}{|c|c|c|c|c|c|c|c|c|}
\hline hote_client & login_client & utilisateur_clien & date_et_heure & methode & |url_des_pages & protocole & |code_de_retour & $\mathrm{dc}$ \\
\hline 127.0 .0 .1 & - & - & $1 / 200501: 25: 09$ & GET & /uh2 $\mathrm{m} /$ & HTTP/1.1" & 200 & 3 \\
\hline 127.0 .0 .1 & - & - & $1 / 200501: 25: 23$ & GET & 7 & HTTP/1.1" & 200 & 2 \\
\hline 127.0 .0 .1 & - & - & $1 / 200501: 25: 23$ & GET & /images_easyp & HTTP/1.1" & 304 & \\
\hline 127.0 .0 .1 & - & - & $1 / 2005$ 01:25:23 & GET & /images_easyp & HTTP/1.1" & 304 & - \\
\hline 127.0.0.1 & - & - & $1 / 200501: 25: 23$ & GET & /images_easyp & HTTP/1.1" & 304 & - \\
\hline 127.0 .0 .1 & - & - & $1 / 2005$ 01:25:23 & GET & /images_easyp & HTTP/1.1" & 304 & - \\
\hline 127.0.0.1 & - & - & $\mid / 200501: 25: 23$ & GET & /images_easyp & HTTP/1.1" & 304 & . \\
\hline 127.0 .0 .1 & - & - & $1 / 2005$ 01:32:25 & GET & / & HTTP/1.1" & 200 & 2 \\
\hline 127.0 .0 .1 & - & - & $1 / 2005$ 01:32:25 & GET & /images_easyp & HTTP/1.1" & 304 & - \\
\hline 127.0 .0 .1 & - & - & $1 / 2005$ 01:32:25 & GET & /images_easyp & HTTP/1.1" & 304 & - \\
\hline 127.0 .0 .1 & - & - & $1 / 200501: 32: 25$ & GET & /images_easyp & HTTP/1.1" & 304 & - \\
\hline 127.0 .0 .1 & - & - & $1 / 2005$ 01:32:25 & GET & /images_easyp & HTTP/1.1" & 304 & - \\
\hline 127.0 .0 .1 & - & - & $1 / 2005$ 01:32:25 & GET & /images_easyp & HTTP/1.1" & 304 & - \\
\hline 127.0 .0 .1 & - & - & $1 / 200501: 34: 20$ & GET & / & HTTP/1.1" & 200 & 2 \\
\hline 127.0 .0 .1 & - & - & $1 / 2005$ 01:34:20 & GET & /images_easyp & HTTP/1.1" & 304 & - \\
\hline 127.0 .0 .1 & - & - & $1 / 200501: 34: 20$ & GET & /images_easyp & HTTP/1.1" & 304 & - \\
\hline 127.0 .0 .1 & - & - & $1 / 2005$ 01:34:20 & GET & fimages_easyp & HTTP/1.1" & 304 & - \\
\hline 127.0 .0 .1 & - & - & $1 / 2005$ 01:34:20 & GET & /images_easyp & HTTP/1.1" & 304 & - \\
\hline 127.0 .0 .1 & - & - & $\mid / 2005$ 01:34:20 & GET & /images_easyp & HTTP/1.1" & 304 & - \\
\hline 127.0 .0 .1 & - & - & I/2005 01:35:18 & GET & $/ \mathrm{uh} 2 \mathrm{~m} /$ & HTTP/1.1" & 200 & 3 \\
\hline 127.0 .0 .1 & - & - & $1 / 200501: 36: 16$ & GET & $/$ uh $2 \mathrm{~m} /$ & HTTP/1.1" & 200 & 3 \\
\hline 127.0 .0 .1 & - & - & $1 / 200501: 36: 29$ & GET & /uh $2 \mathrm{~m} /$ & HTTP/1.1" & 200 & 3 \\
\hline 127.0 .0 .1 & - & - & $1 / 2005$ 01:36:30 & GET & /uh $2 \mathrm{~m} /$ & HTTP/1.1" & 200 & 3 \\
\hline 127.0 .0 .1 & - & - & $1 / 200501: 36: 36$ & GET & /uh $2 \mathrm{~m} /$ & HTTP/1.1" & 200 & \\
\hline 127.0 .0 .1 & - & - & $1 / 200501: 36: 54$ & GET & /uh $2 \mathrm{~m} /$ & HTTP/1.1" & 200 & $3+$ \\
\hline
\end{tabular}

Figure 7 : Base de données après import

\section{3-2. Nettoyage des données}

\section{3-2-1. Nettoyage des graphiques, images ou scripts}

Les données concernant les pages possédant des graphiques, des images ou des scripts, n'apportent rien à l'analyse. Elles seront donc filtrées et éliminées.

Pour cela on est amené à supprimer de notre base de données les URLs qui ont les formes suivantes:

Les urls correspondant aux images d'extension II.gifl par la requête

("delete from LOGUNIV where urI_des_pages like "\%.gif\%")

Les urls correspondant aux images d'extension II.jpg" par la requête

("delete from LOGUNIV where url_des_pages like '\%.jpg\%")

Les urls correspondant aux images d'extension II.icoll par la requête

("delete from LOGUNIV where url_des_pages like "\%.ico")

Les urls correspondant aux feuilles de styles d'extension II.cssil par la requête

("delete from LOGUNIV where url_des_pages like '\%.css")

Les urls correspondant aux images d'extension II.pngll par la requête

("delete from LOGUNIV where url_des_pages like '\%.png")

\section{3-2-2. Résolution des pages uniques interprétées différemment par l'interpréteur du serveur}

Pour les pages qui ont des extensions II.phpı, on doit résoudre le problème de ॥ la page unique qui est interprétée différemment II par l'interpréteur du serveur. 


\begin{tabular}{|c|c|c|c|c|c|c|}
\hline login_client & |utilisateur_clien & date_et_heure & url_des_pages & protocole & |code_de_erreul & \\
\hline- & - & $/ 2006$ 02:21:45 & /chimie $\mathrm{htm}$ & HTTP/1.1" & 200 & \\
\hline- & - & $/ 2006$ 02:29:00 & /robots.txt & HTTP/1.0" & 404 & $\infty$ \\
\hline- & - & $/ 2006$ 02:36:3 & /forum/read. php?f=3\&i=272\&t=2 & HTTP/1.1" & 200 & \\
\hline- & - & $/ 2006$ 02:38:2 & /forum/read php?f=3\&i=589\&t=2 & HTTP/1.1" & 200 & \\
\hline- & - & $/ 2006$ 02:38:3 & /forum $/$ read php? $=3 \& \mathrm{i}=618 \& \mathrm{t}=2$ & HTTP/1.1" & 200 & \\
\hline- & - & $/ 2006$ 02:42:4 & /forum/read. php?f=3\&i=292\&t=2 & HTTP/1.0" & 200 & \\
\hline- & - & $/ 2006$ 02:42:4 & /forum/read. php?f=3\&i=201\&t=2 & HTTP/1.0" & 200 & \\
\hline- & - & $/ 200602: 42: 4$ & $/$ forum $/$ read. php?f $=3 \& t=2 \& a=2$ & HTTP/1.0" & 302 & \\
\hline- & - & $/ 2006$ 02:42:4 & /forum $/$ read php? $=3 \& \mathrm{i}=1336 \& \mathrm{t}=1336$ & HTTP/1.0" & 200 & \\
\hline- & - & $/ 2006$ 02:42:4 & $/$ forum/read. php?f=3\&i=890\&t=2 & HTTP/1.1" & 200 & \\
\hline- & - & $/ 200602: 42: 5$ & /forum/read. php?f=3\&i=201\&t=2 & HTTP/1.0" & 200 & \\
\hline- & - & $/ 2006$ 02:51:12 & /menu.htm & HTTP/1.1" & 200 & \\
\hline- & - & $/ 200602: 51: 12$ & /menu. swf & HTTP/1.1" & 200 & \\
\hline- & - & $/ 2006$ 02:51:12 & /main.php & HTTP/1.1" & 200 & \\
\hline- & - & $/ 2006$ 02:51:31 & /formation.htm & HTTP/1.1" & 200 & 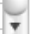 \\
\hline$\because$ (14) & $\overline{1}(\bullet)(1) *$ & sur 1525 & $40 \Longleftarrow$ & $-\cdots \cdot \cdots$ & -- & \\
\hline
\end{tabular}

Figure 8 : Pages uniques interprétées différemment par le serveur

Alors l'idée est de modifier la colonne II url_des_pages ॥ de telle sorte à supprimer pour une même page, la partie de son adresse url, qui commence par ॥?" jusqu'à la fin de l'url.

Par exemple, couper la partie de l'url, qui suit le ॥?" jusqu'à la fin de la ligne, et ensuite la supprimer :

$$
\text { /forum/read.php | | ? f=3\& } \mathrm{i}=940 \& \mathrm{t}=625 \text { | | | }
$$

L'algorithme utilisé pour cette modification est le suivant :

\begin{tabular}{|l|}
\hline ch est une chaîne \\
m est un entier \\
y est un entier \\
POUR m=1 A Table2..Occurrence \\
ch est une chaîne \\
ch est un entier \\
ch=Table2.Pages[m] \\
y=Position(ch,"?",DepuisDébut) \\
SI y<>0 ALORS \\
ch=ExtraitChaîne(ch,l,"?") \\
Table2.Pages[m]=ch \\
FIN \\
\hline
\end{tabular}




\section{3-2-3. Résolution du Problème du Format Date et Heure}

L'un des problèmes à résoudre aussi, est le format des champs II date_et_heure II du fichier $L O G$ qu'on utilise pour garder la trace de l'instant d'entrée de l'internaute. Ce format est incompatible avec celui utilisé dans la base de données (Figure 8).

Le format adéquat pour ce champ est II date_et_heure II.

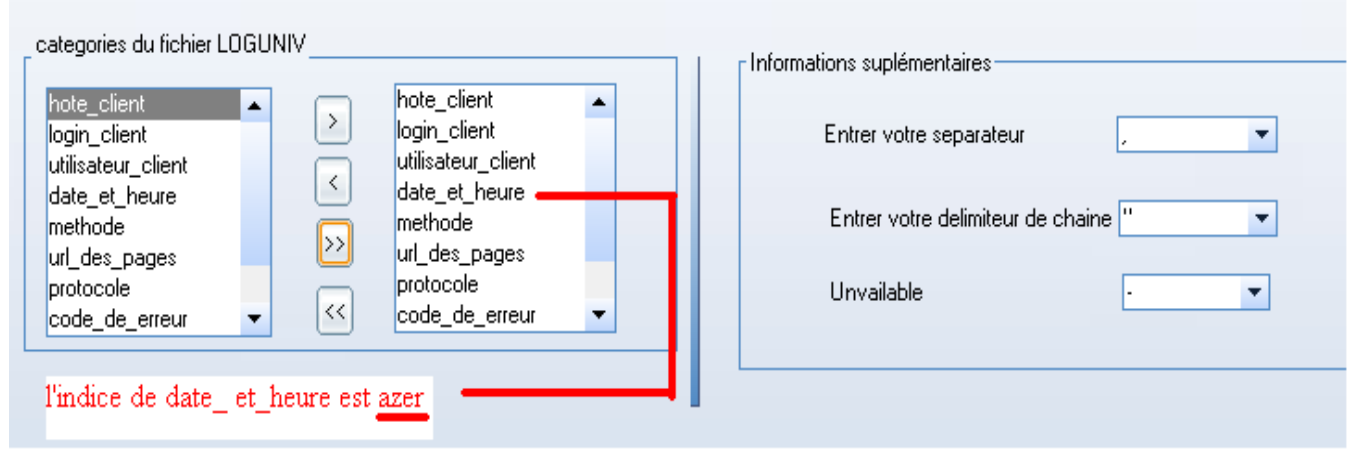

Figure 9 : Format date et heure

L'algorithme utilisé pour rendre la date et l'heure du fichier $L O G$ reconnaissable par la colonne II date_et_heure II du SGBD est comme suit :

On commence par repérer la position courante de la colonne II date_et_heure II dans la liste de catégorie du fichier $L O G$, qui nous renseigne sur son emplacement dans le fichier LOG (Figure 9).

\section{Algorithme}

\begin{tabular}{|c|}
\hline $\begin{array}{l}\text { BOUCLE } \\
\text { Ch est chaine } \\
\text { ch=flitligne(id) } \\
\text { SI ch = EOTALORS SORTIR } \\
\text { chl est une chaîne="'" } \\
\text { chl1 est une chaîne="' } \\
\text { ch12 est une chaîne } \\
\text { ch13 est une chaîne } \\
\text { h est un entier } \\
\text { h=azer+l } \\
\text { //azer est l'indice de la colonne date_et_heure dans la liste } \\
\text { POUR i=l A nbre } \\
\text { chll=ExtraitChaîne(ch,azer,sep) } \\
\text { chl2=ExtraitChaîne(chll,l,":") }\end{array}$ \\
\hline
\end{tabular}




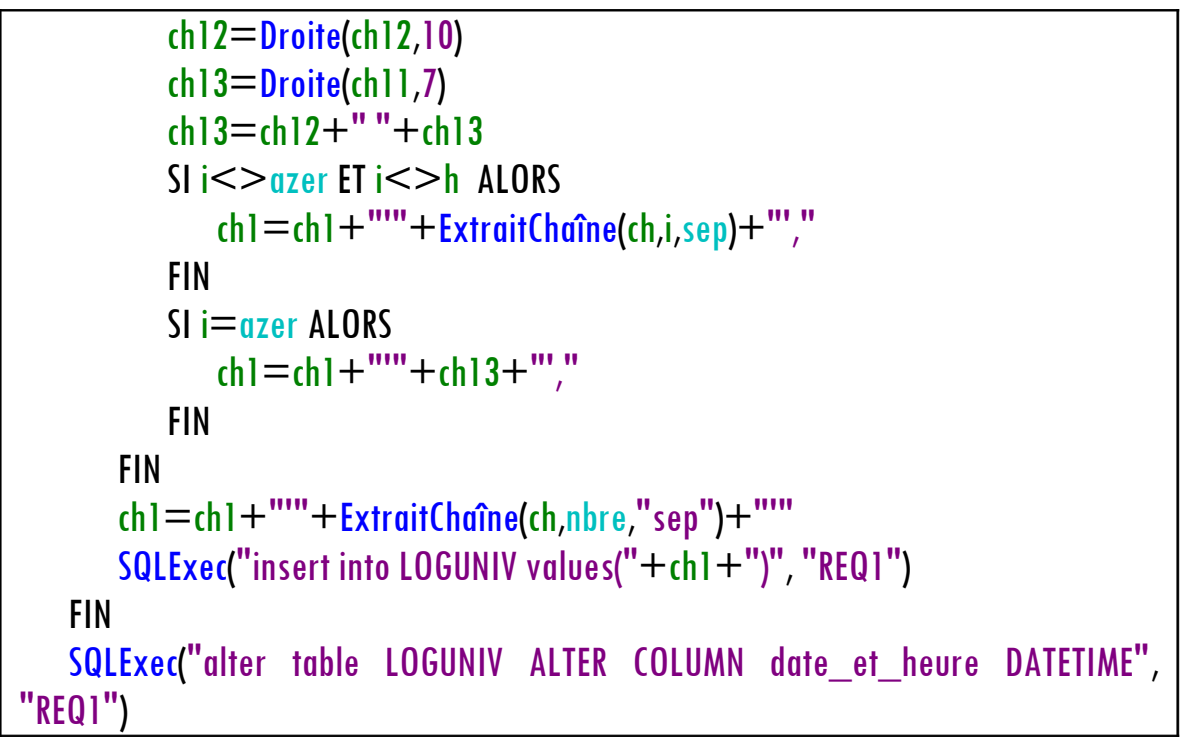

Après ces quatre grandes étapes de pré-traitement et nettoyage, le fichier Log est enfin prêt pour l'exploration et l'analyse.

\section{Exploration et analyse du fichier $\log$}

Pour l'exploration et l'analyse du fichier $\log$, un outil logiciel a été conçu et réalisé : " LOG ANALYZER ॥, dont l'interface est comme suit :

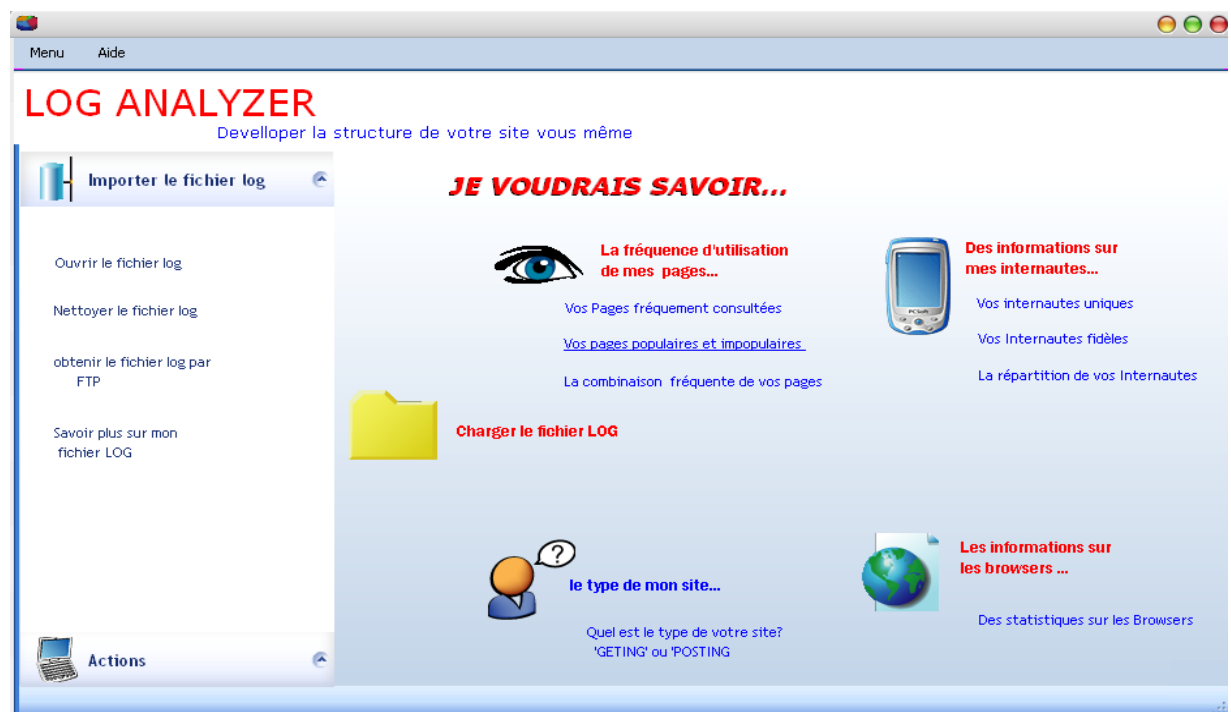

Figure 10 : Interface du logiciel " LOG ANAL YZER ॥ 


\section{4-1. Les combinaisons fréquentes des pages}

Pour déterminer la combinaison des pages les plus utilisées, on a utilisé la méthode ॥ $\boldsymbol{A}$ Priori II.

On commencera tout d'abord par définir quelques notions utilisées dans cette méthode :

- Nombre de pages : c'est le nombre total des pages dans le fichier Log (dans la base de données)

- Une session : peut être soit une adresse IP, soit une demi-heure si l'adresse IP se répète pour une durée supérieure à une demi-heure.

- Support d'une page : c'est la somme du nombre d'apparition de la page dans toutes les sessions, divisée par le nombre total des sessions.

- Support minimum : seuil de support.

- Page fréquente : c'est la page dont le support est supérieur au support minimum.

- Les combinaisons des pages: elles sont déduites en utilisant l'algorithme "I A Priori ॥

\section{L'algorithme A Priori - 2}

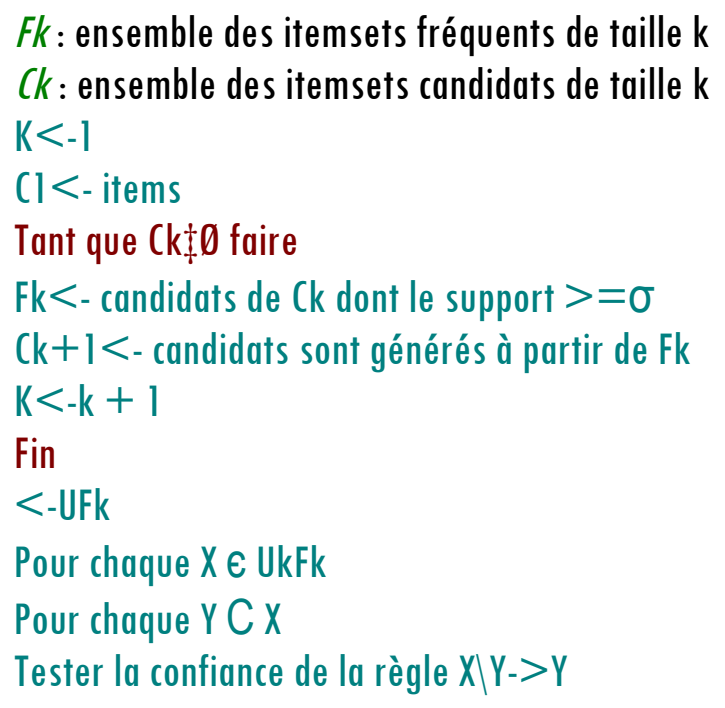

Fin

$<-$ UFk

Pour chaque $X \in U \mathrm{UFk}$

Pour chaque Y $C X$

Tester la confiance de la règle $X \mid Y->Y$

\section{4-2. Les pages fréquemment consultées}

On doit spécifier, tout d'abord, la période pour laquelle on aimerait faire la consultation (Figure 11), puis on calcule, pour chaque page, la fréquence de consultation. 


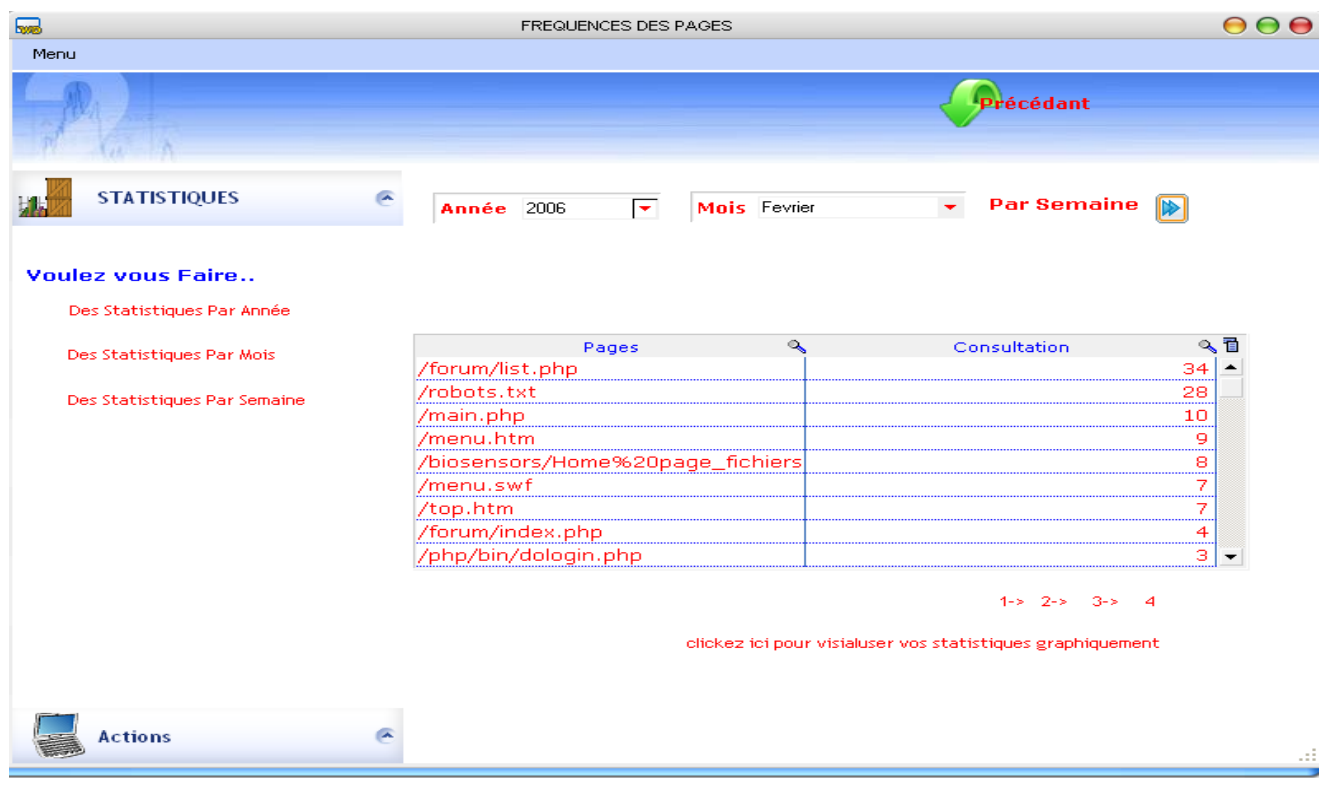

Figure 11 : Pages fréquemment consultées?

On peut l'illustrer graphiquement comme suit :

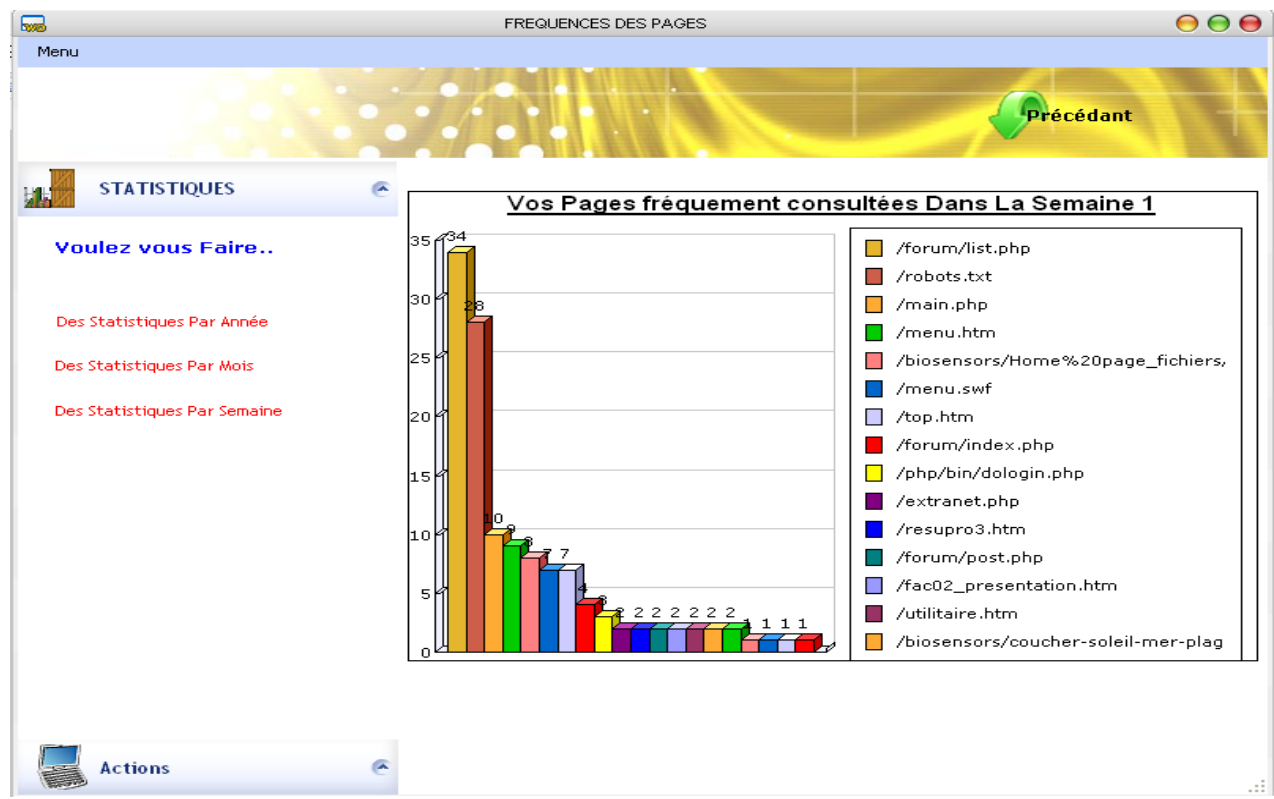

Figure 12 : Graphique des pages fréquemment consultées 


\section{4-3. Pages populaires et impopulaires}

On doit choisir la période pour laquelle on voudrait faire la consultation. Un tableau de statistiques sur les pages populaires et impopulaires selon la période spécifiée sera affiché (Figure 13).

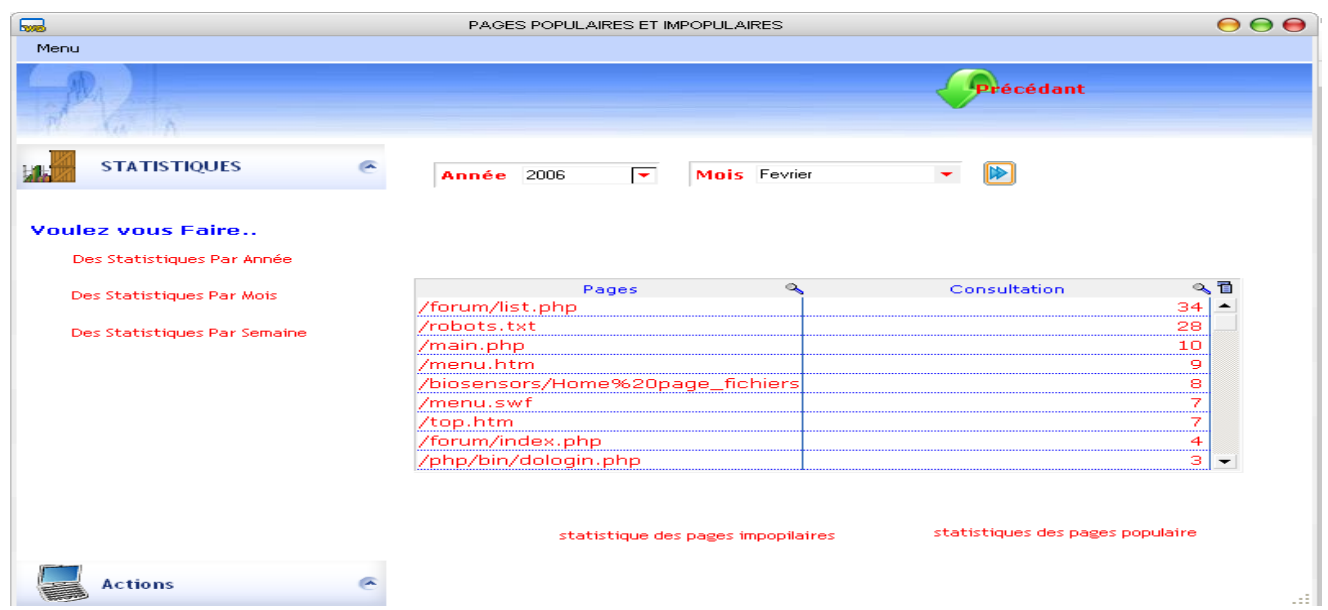

Figure 13 : Pages populaires et impopulaires

Ici l'idée est de donner au Webmaster toutes les pages populaires et impopulaires selon leurs degrés de popularité, en se basant sur le nombre de fois qu'une page a été visitée dans une période spécifiée $[7,8]$.

Graphiquement, on obtient ceci à la Figure 14 :

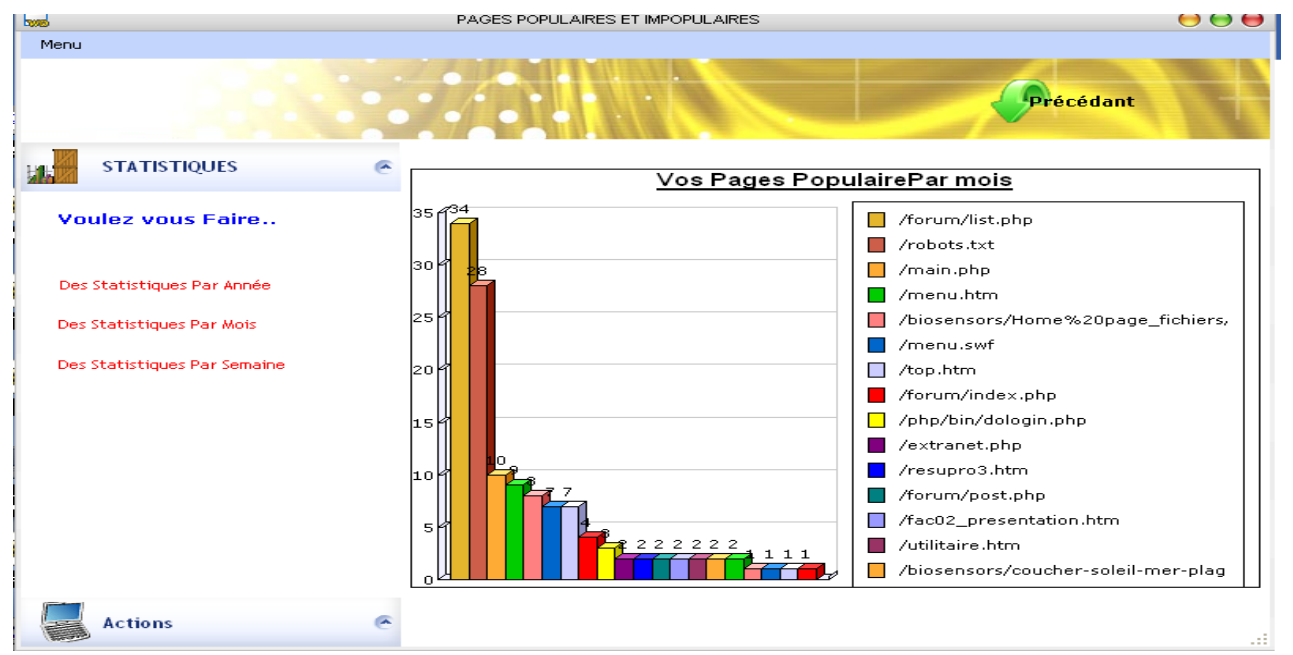

Figure 14 : Graphique des pages populaires et impopulaire 


\section{4-4. Informations sur les internautes}

Cette rubrique renseigne le Webmaster sur la fidélité de ses visiteurs (Figure 15).

NB : chaque adresse IP est équivalente à un utilisateur ou internaute.

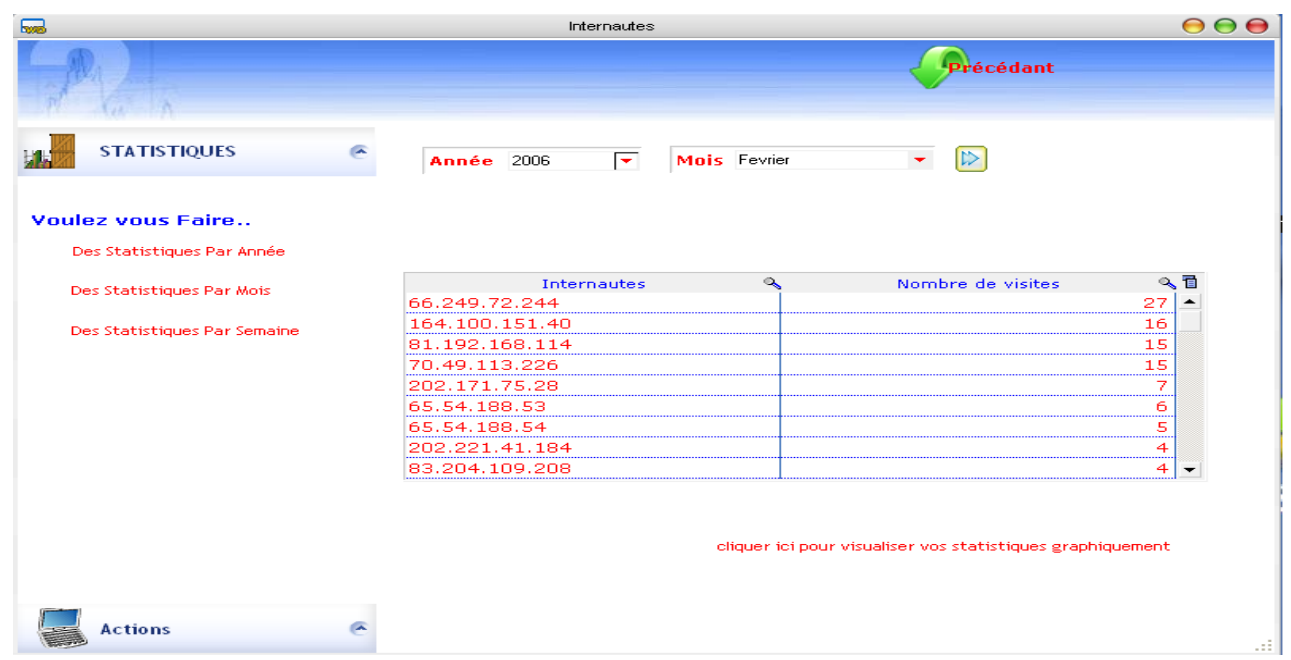

Figure 15 : Informations sur les internautes.

\section{4-5. Catégorie du site}

Dans cette rubrique on déterminera si notre site joue toujours son rôle préalablement défini (consultatif ou de téléchargement), en utilisant les méthodes sur le site (GET OU POST).

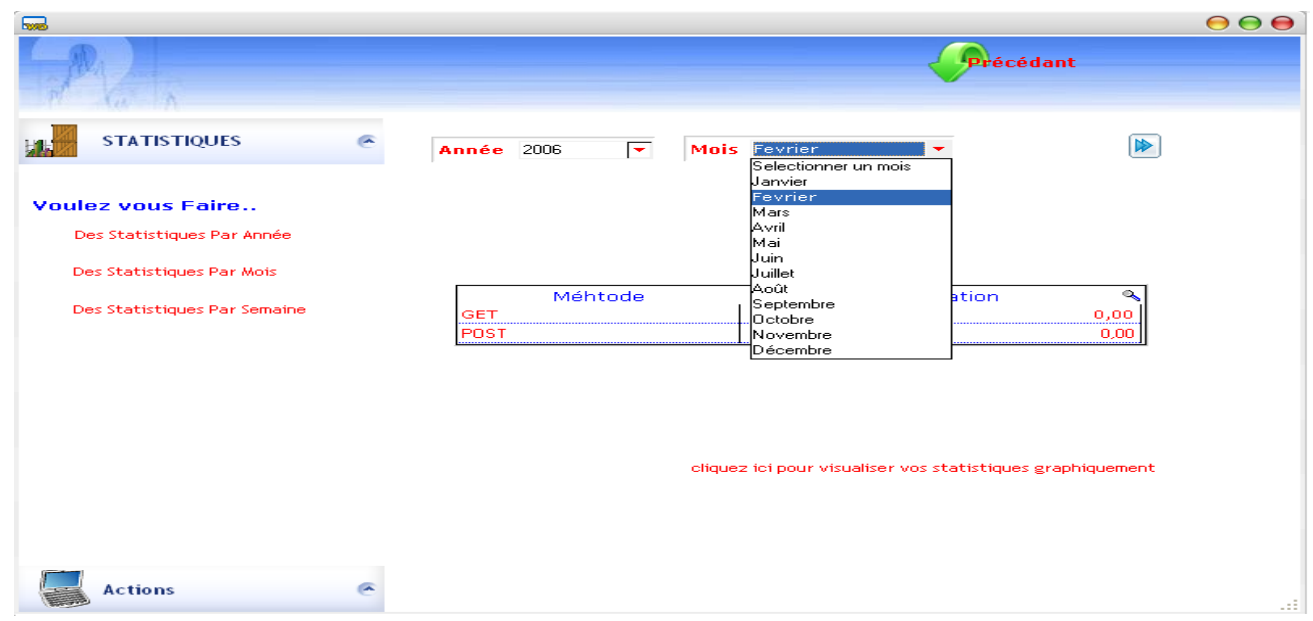

Figure 16 : Catégorie du site 
Parfois, un site défini préalablement comme consultatif avec quelques fichiers à télécharger se transforme en un site de téléchargement, car les utilisateurs ne le consultent que pour les téléchargements. Par conséquent, ce site ne satisfait pas ses utilisateurs comme son Webmaster l'avait prévu. On peut l'illustrer graphiquement par la Figure 17:

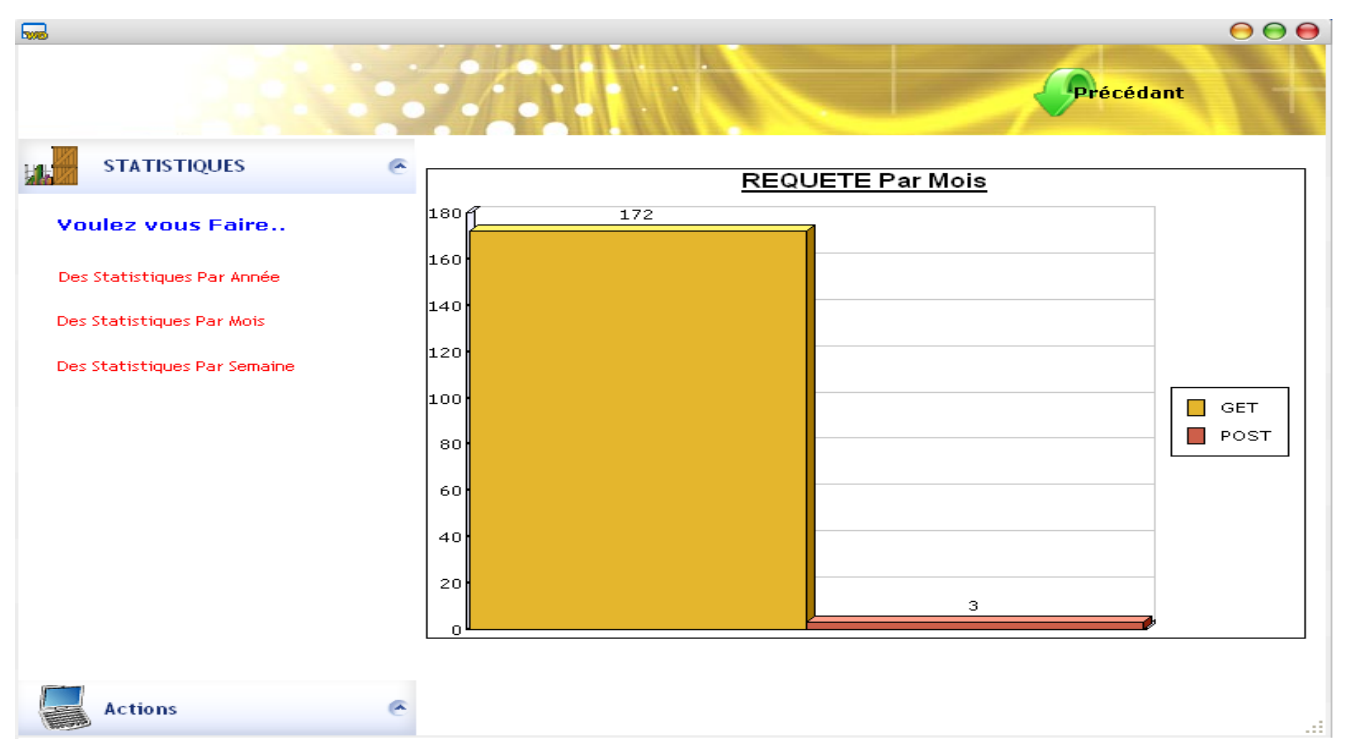

Figure 17 : Graphique sur la catégorie du site

\section{4-6. Types de navigateurs (Browsers)}

Si on clique sur le lien "I statistiques sur les browsers II, une page nous demande de choisir la période pour laquelle on aimerait afficher un diagramme résumant les pourcentages d'utilisation des browsers pour accéder au site :

NB : seuls les navigateurs "célèbres" sont pris en compte 


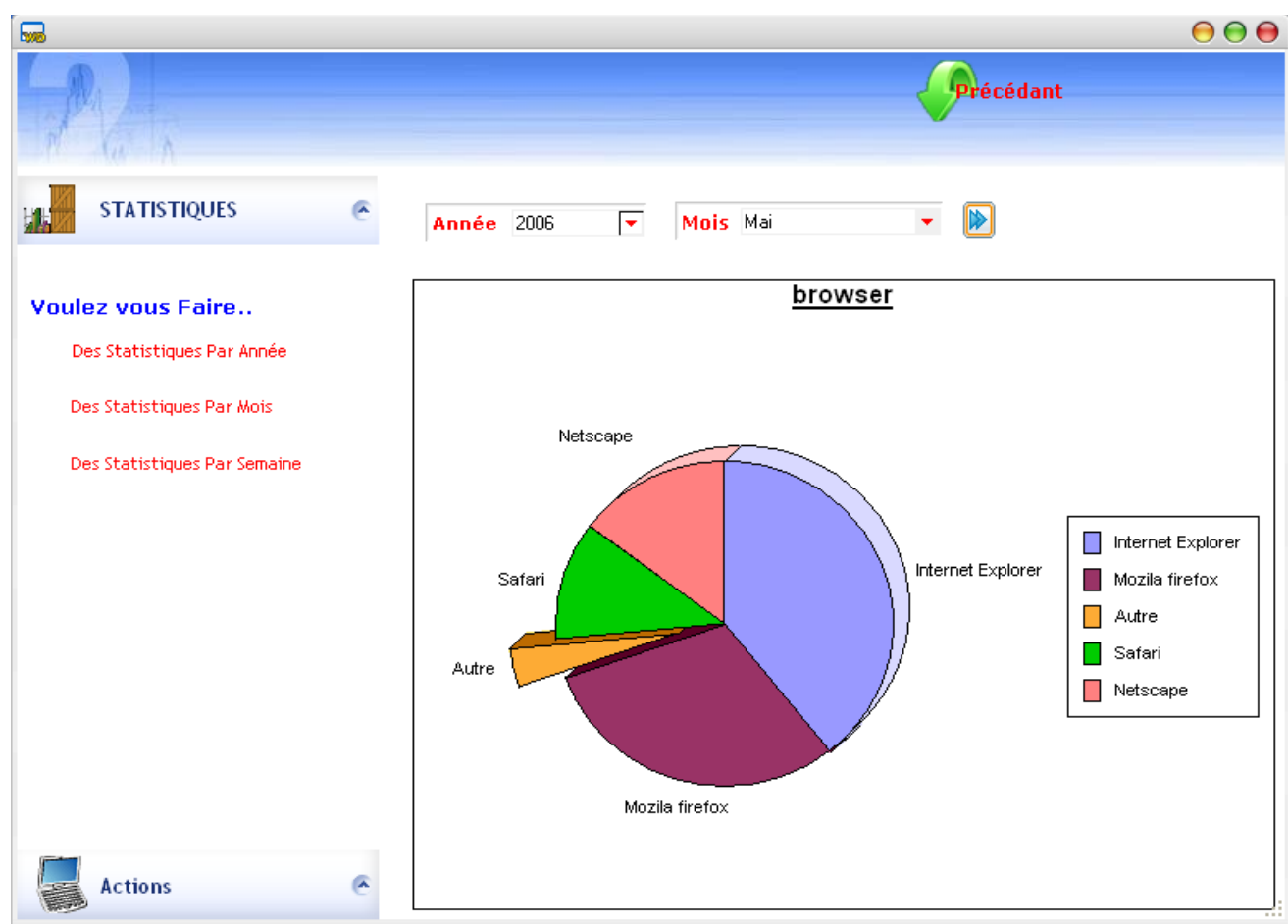

Figure 18 : Types de navigateurs

\section{Conclusion et perspectives}

Dans ce travail, nous avons montré comment les techniques du Web Usage Mining peuvent contribuer à l'amélioration et au diagnostic de site Web, sur un cas concret : le site de I'Université Hassan II-Mohammedia : www.univh2m.ac.ma, en explorant le fichier $\log$ du serveur Web de ladite Université.

Ce travail est toujours en cours d'amélioration, en ce qui concerne les thèmes abordés et les fonctionnalités. On focalisera notre intérêt sur le " profil du visiteur "l et de " groupe de visiteurs ", et on enrichira le travail par l'association d'une " cartographie " des visiteurs. 


\section{Références}

[1] - Site http://www.internetworldstats.com/stats.htm, "world internet usage and population statistics", http://www.netcraft.com, December 2006, Web Server Survey.

[2] - R. Rao, P. Pirolli, J. Pitkow. "Silk from a sow's ear : extracting usable structures from the web". Human Factors in Computing Systems", CHI. (1996). in Proceeding of ACM Conference on Human Factors in Computing Systems, Vancouver, British Columbia , Canada, April 13-18 (1996). pages 118-125. http://www.sigchi.org/chi96/proceedings/papers/Pirolli_2/pp2.htm/

[3] - B. Mobasher, H. Dai, T. Lou, et M. Nakagawa "Discovery and evaluation of aggregate usage profiles for web personalization", Data Mining and Knowledge Discovery, Vol. 6, $\mathrm{N}^{\circ} 1$ (2002), Jan., pages 61-82.

[4] - H. Azzag, C. Guinot, G. Venturini, II Classification hiérarchique et visualisation de pages Web II, Actes de l'atelier Fouille du Web des $6^{\text {èmes }}$ journées francophones, "I Extraction et Gestion des Connaissances EGC 2006 II. ENIC Telecom Lillel, Cité Scientifique, Lille, France. 17-20 Janvier (2006) pages 5-16.

[5] - R. Cooley, B. Mobasher, and J. Srivastava, II Data Preparation for Mining World Wide Web Browsing Patterns II, J. Knowledge and Information Systems, Vol. I, $\mathrm{N}^{\circ} 1$ (1999). pages 5-32.

[6] - M. Charrad, M. Ben Ahmed et Y. Lechevallier II Web Usage Mining: WWW pages classification from log files 11 . Actes de l'atelier Fouille du Web des 6èmes journées francophones, "I Extraction et Gestion des Connaissances EGC 2006 II à ENIC Telecom Lille 1, Cité Scientifique Lille, France. 17-20 Janvier (2006) pages 41-52.

[7] - Y. Lechevallier, D. Tonasa, B. Trousse, R. Verde. "Classification automatique : Applications au Web Mining 11. in Proceeding of SFC 2003, Neuchatel, Swiss. Septembre (2003) pages 10-12.

[8] - M. Charrad, II Techniques d'extraction des connaissances appliquées aux données du Web II. Mémoire de Mastère présenté en vue de l'obtention du diplôme de Mastère en Informatique, Ecole Nationale des Sciences de l'Informatique de Tunis, Laboratoire RIADI (2005).

[9] - R. Kimball et R. Merz II Le data webhouse. Analyse des comportements clients sur le Web II. Editions Eyrolles, Paris (2000). 